\title{
Study on Computer Information Processing Technology in the Era of Big Data
}

\author{
Liu Jian \\ Tianjin Metallurgical Technical and Vocational College
}

(China Tianjin300350)

Keywords: Computer; Big data; Information technology

\begin{abstract}
With the progress of science and technology, computer technology has been widely used in the world, and with the advent of the era of big data, establishing and improving the computer information processing technology has become the main direction of the development of computer technology at present. This paper studies the background of big data era, and the computer information processing technology is studied and discussed in detail to provide theoretical support for the development of computer information technology in the era of big data.

With the rapid development of computer technology and network technology, people's life, work and medical care are gradually entering the era of network, and people begin to enter the era of big data. The application of computer technology is the core of the era of big data, so gradually improve the computer hardware facilities to ensure that the computer network technology and computer information processing technology can easily grasp the initiative in the era of big data with a leading position in various fields. Therefore, based on the original application of computer and network technology, developing and improving the computer information processing technology is of great significance to enhance the level of development of various industries in China and enhance the power status
\end{abstract}

\section{Part One Definition and characteristics of "big data"}

Definition of "big data" . "Big data" is the result of social development, the progress of science and technology and the industry of social data since twenty-first Century, its main content is with the use of computer technology to record the life and work bit by bit, so as to achieve rule-based, auditable level. Through the investigation of the current computer hardware and software facilities, it is found that the current computer, whether it is data storage function or data processing function can not meet the requirements of real-time and accurate recording of large amounts of data. "Big data" is mainly through a large number of continuous accumulation and contrast of data to quickly get a product production methods, as well as get person's life trajectory and other functions. In the current situation, with the people's daily flow of high demand for news and video data, the production of computer and software company also gradually to improve the personal computer and the large computing power through the upgrading of products and product upgrades and other means.

Characteristics of "big data" . When study the computer information processing technology in the era of big data, we should first have a comprehensive understanding for the characteristics of the era of big data information in order to improve the computer information processing technology and to meet the big data era's needs of computer information processing technology. Characteristics of "big data" information is divided into the following three aspects: the first is characterized by a large amount of data, due to the realization of all industries, big data of our life must be on the process of various industries with the various products of the production process, and it will result in the consequences of record action track which is the record of everyone's life in all the time, this is not a simple work here that highlights the big data; the second characteristic is that the data transmission speed is fast, because of a large amount of data so it requires the collection of information must be transferred to a stable and fast reliable storage space by transmission, which can ensure data integrity and reliability and can also prevent the destruction of 
data; the third characteristic is more information, with the rapid development of various industries, in order to achieve the real meaning of the "big data era", it is bound to track and record all relevant information which will lead to the diversified development of information.

\section{Part Two the key link of information processing technology in the era of big data}

Collection and transmission of information. The main form of big data is the data information, to achieve all walks of life, every field must carry on the data collection and data transmission, while post processing is based on the previous data acquisition. In order to realize the acquisition of information, it has the following main steps: firstly, select required target data, and then through the real-time monitoring of the operation of the track to get the required data, and through the relevant software to be transferred to the storage space in a particular; secondly, to achieve accurate search data, people must first finish certain standard storage and storage arrangement according to the data; finally, after the completion of data collection, before data is in a specific storage space for transmission and storage, it needs to determine the good transmission ways and means in advance to ensure the integrity of the information.

Storage of data information. Data storage is mainly refers to the information collected through specific channels transmitted back to the storage location, these data will be stored in a specific computer database to facilitate later users can search through simple query function, the process for data storage needs on the higher only the completion of the data storage's stability on information database and searching, and it can realize the user access to information and data processing operations. Therefore, the storage of computer information in the era of big data is of great significance for all walks of life to achieve big data.

Information security processing. To ensure the integrity, accuracy and safety of the data, it must have safe handling of the relevant information, the main means for the big data era of computer information processing has the following two aspects: one is to establish a computer information security department, perfect the management mechanism, and the mechanism of regular maintenance and upgrade to ensure the safety of information; the other one is to increase investment for large data processing software development of information security to ensure the safety of information processing in software. Therefore, in the information collection and storage, it needs for information security measures in order to ensure the safety and integrity of data information.

\section{Part Three Computer information processing technology in the "era of big data"}

Data collection and dissemination technology. As everyone knows, what we see on TV and Internet video and audio heard are processed in post production and dissemination of the information processing and molding process, which is to ensure the integrity of the information or reach a certain that is deemed to have effect, and it must be processed by professionals in all aspects after a period of communication process. Based on the research of data collection and communication technology that the era of big data processing technology of computer information is mainly composed of information collection, sorting and screening of late prophase and further processing. As the first step of data processing, data collection and communication occupies a very important position in the computer information processing technology, we should increase research and the importance of investment on the data collection and communication technology. Therefore, in the era of big data, computer practitioners and professionals should pay attention to the the research on computer data collection speed, precision and transmission speed and transmission quality in order to prepare for the post of computer information processing.

Information storage technology. As for computer, information storage as one of its most basic functions plays an important role in the application of computer. In the era of big data, the kind of information people come into contact with and quantity are very large, but also to achieve the real big data, it is necessary to store large amounts of data, to prepare for late in the screening and processing of information. With the rapid development of computer technology, people put forward 
higher requirements for the computer storage space, which to some extent to meet the computer storage requirements, and provides unlimited storage space for the era of big data with data information in computer memory to facilitate people's life and work.

In recent years, the computer has gradually become the main office equipment, a computer will have an important impact on the quality of people's life and work, as the computer storage technology directly determines the computer running speed and storage space of the computer information technology with more and more functions. But with people's production and daily life for the urgent needs of big data, the computer needs to have more storage space to record the daily life trajectory of each product process and design ideas as well as people. Therefore, only by constantly improving the computer storage technology, can we calmly deal with computer information of the big data era for the high requirements of computer storage space.

Information security technology. On the computer, people can realize the receiving and processing of various data only in the case of networking, and security risks and vulnerabilities inside the computer system and computer network exist on information security and integrity with the great influence. Therefore, the continuous improvement and application of computer information security technology is of great significance to ensure the safety and integrity of computer information in the era of big data. At present, the information security technology used is the correlation between various data based on the data, once there is a link error, it must be verified and tested all the information relating to the link which is the only way to fully ensure the security of computer information. With the arrival of the era of big data, due to the limitations of computer hardware and software facilities, as well as computer processing speed and other reasons, it will lead to serious threats to computer information security.

In order to cope with the security of computer information in the era of big data, we should make sure that the computer security system should be improved from the aspects of computer hardware, software and computer system. For computer professionals, they should strengthen education and training, and vigorously develop computer network security and information security software from multiple aspects to ensure the security of computer information in the era of big data. To sum up, with the significance of computer information security for big data era on computer security and integrity of information, as employees, we should increase the intensity of the development of computer information security, and gradually improve relevant personnel on attention and awareness for computer information security degree.

Information processing and transmission technology. For large data processing of computer information is not a simple information sorting and classification work, but it needs to be detailed information screening and meticulous processing to achieve. The information processing and transmission technology is mainly divided into the following three parts: firstly, the best screening of source of information, and it is namely to the sources of information are identified to ensure the legitimacy of the source of information, then the legal information is stored in the corresponding database; secondly, according to the selection of information processing in detail and the information processing is mainly in accordance with the classification and properties separately; finally, the information will be processed according to the categories and characteristics of the corresponding classification and transmission to the client, so that customers can query and review for data.

\section{Part Four Conclusion}

With the advent of the era of big data, huge data acquisition, transmission, processing and storage with great pressure and challenge to the computer industry, these pressures and challenges are also constantly promote and inspire the development of the computer information processing technology. Computer information processing technology will gradually improve to human production and bring great convenience to life, believe that in the future, we will be able to rely on the perfect computer information processing technology and computer personnel professional level can guide humanity to a new journey. 


\section{Reference}

[1] Jiahui Niu.Analysis of computer information processing technology in the era of big data[J].Computer Fan, 2017(1): 1.

[2] Guanliang Zhan.Analysis of computer information processing technology in the era of "big data”[J].Scientific and Technological Wind, 2017(19): 59.

[3] Rui Sun.Analysis of computer information processing technology in the era of big data[J].Technology Outlook, 2016(26): 11. 